\title{
(2) OPEN ACCESS \\ MicroRNA-363-3p downregulation in papillary thyroid cancer inhibits tumor progression by targeting NOB1
}

\author{
Su Dong, ${ }^{1}$ Shuai Xue, ${ }^{2}$ Yue Sun, ${ }^{3}$ Zhe Han, ${ }^{2}$ Lele Sun, ${ }^{2}$ Jialu Xu, ${ }^{2}$ Jia Liu (i) ${ }^{2}$
}

Additional material is published online only. To view please visit the journal online (http://dx.doi.org/10 1136/jim-2020-001562)

${ }^{1}$ Anesthesia, First Hospital of Jilin University, Changchun, China

${ }^{2}$ Thyroid Surgery, First Hospital of Jilin University, Changchun, China

${ }^{3}$ First Operating Room, First Hospital of Jilin University, Changchun, China

Correspondence to Professor Jia Liu, Thyroid Surgery, First Hospital of Jilin University, Changchun, China; sosea@sina.com

Accepted 24 September 2020

Published Online First 19 October 2020

\begin{abstract}
MicroRNA-363-3p (miR-363-3p) has been reported to play a crucial role in tumor development and progression, and function as a tumor suppressor in many types of cancer. In our previous studies, we found that miRNA-363-3p inhibited papillary thyroid carcinoma (PTC) progression by targeting PIK3CA. Meanwhile, we found that NIN1/RPN12 binding protein 1 (NOB1) was significantly upregulated in thyroid carcinoma tissue and downregulation of NOB1 expression significantly inhibited cell proliferation, migration and invasion in PTC. However, the correlation of NOB 1 and miR-363-3p has not been investigated. Here, we performed bioinformatic analysis to explore miRNA targeting NOB1. We found that NOB1 was a target of miR-363-3 $p$ and $\mathrm{miR}-363-3 p$ regulated NOB1 expression at the translational and transcriptional levels by targeting its $3^{\prime}$ untranslated region ( $3^{\prime}$-UTR). Further, we showed that miR-363-3p inhibited tumor progression by targeting NOB1 in vitro and in vivo. We found that overexpression miR363-3 $p$ or silencing NOB1 significantly increased G0/G1-phase and decreased S-phase in the human papillary thyroid cells, which led to a significant delay in cell proliferation, indicating miR-363-3p and NOB 1 are crucial for human papillary thyroid cancer tumorigenesis. Collectively, our data unveil that miR-363-3 p negatively regulates NOB1 activity by reducing its stability. This study provides a new therapeutic target for regulation of NOB1 stability to modulate human papillary thyroid cancer progression.
\end{abstract}

\section{INTRODUCTION}

Papillary thyroid carcinoma (PTC) incidence has increased substantially in the world over the last several decades. ${ }^{1}$ Most patients with PTC have an expected favorable prognosis after surgical resection combined with radioiodine and levothyroxine treatment. ${ }^{2}$ However, the incidence rate and mortality rate for advancedstage papillary thyroid cancer increased in recent years. In the cases of metastasis, patients had poorer responses to standard therapies than localized PTC. ${ }^{3}$ The understanding of the molecular mechanisms of progression in PTC plays a critical role in predicting clinical outcomes and is beneficial to the targeted therapies.

\section{Significance of this study}

What is already known about this subject?

- MicroRNA-363-3p (miR-363-3p) functions as a tumor suppressor.

- miR-363-3 p downregulates in papillary thyroid cancer cell lines and tissues.

- miR-363-3 $p$ is negatively associated with clinical stage and lymph node metastasis.

- miR-363-3p inhibits papillary thyroid cancer cell proliferation, migration and invasion targeting PIK3CA.

What are the new findings?

- miR-363-3p targets NOB1.

- miR-363-3 $p$ negatively regulates NOB1 in papillary thyroid cancer.

- miR-363-3 $p$ inhibits papillary thyroid cancer proliferation, migration and invasion by directly targeting NOB1.

How might these results change the focus of research or clinical practice?

- Our findings suggest that exogenous overexpression of miR-363-3p may be a potential drug candidate for the treatment of papillary thyroid carcinoma.

MicroRNAs (miRNAs) are a class of endogenous non-coding RNA molecules, which are involved in regulating almost all biological processes, including cell proliferation, differentiation, metabolism and immune responses. ${ }^{4-6}$ MiRNA molecules have been shown to function as regulators of gene expression, tumor suppressor genes (antimetastatic) and oncogenes (prometastatic). ${ }^{78}$ Recent studies have shown that several miRNAs were expressed abnormally in serum/plasma/tissue in PTC tumor initiation, progression and aggressiveness. ${ }^{9} 10$ MiR-363-3 p has been reported to function as a tumor suppressor in osteosarcoma, ${ }^{11}$ colorectal cancer, ${ }^{12}$ non-small-cell lung cancer, ${ }^{13}$ breast cancer, ${ }^{14}$ head and neck cancer, ${ }^{15}$ renal cancer, ${ }^{16}$ hepatocellular caner ${ }^{17}$ and neuroblastoma. ${ }^{18}$ However, it functions as an oncogene in prostate cancer ${ }^{19}$ and gastric cancer. ${ }^{20}$ In our previous studies, we found that the expression of miR-363-3 p was downregulated in PTC cell lines and tissues, and was negatively associated 
with clinical stage and lymph node metastasis. ${ }^{21}$ We also found that miR-363-3p overexpression inhibits papillary thyroid cancer cell proliferation, migration and invasion in vitro, as well as suppresses tumor growth in vivo directly targeting PIK3CA. ${ }^{21}$

NIN1/RPN12 binding protein 1 (NOB1) is a subunit of the $26 \mathrm{~S}$ proteasome and plays a crucial role in protease function and RNA metabolism. ${ }^{22}{ }^{23}$ The NOB1 gene, located on chromosome $16 \mathrm{q} 22.1$, encoding a $50 \mathrm{kDa}$ protein consisting of a PilT amino terminus domain and a zinc ribbon domain, is mainly expressed in the liver, lung and spleen. ${ }^{224}$ Enhanced production of NOB1 protein has been reported in many human carcinomas. ${ }^{25-28}$ Our previous studies showed that NOB1 protein and mRNA expression were significantly upregulated in thyroid carcinoma tissue, in comparison with thyroid adenoma and normal thyroid tissues and multinodular goiter tissues. ${ }^{29}$ We also found that downregulation of NOB1 expression significantly inhibited cell proliferation, migration and invasion and induced cell apoptosis in vitro, and suppressed tumor growth in vivo, as well as enhanced the radiosensitivity of PTC cells. ${ }^{29}$ Moreover, our results also showed that downregulation of NOB1 was able to significantly activate constitutive phosphorylation of p38 mitogen-activated protein kinase (MAPK), which might contribute to the inhibition of PTC cell growth. These findings suggest that NOB1 may be a potential therapeutic target for the treatment of PTC. ${ }^{29}$ Adenovirus expression vector carrying NOB1 siRNA ( $\mathrm{ad} /$ sh-NOB1) combined with doxorubicin treatment is a potential drug candidate for the treatment of PTC. ${ }^{29}$

To date, the correlation of NOB1 and miRNA-363-3p has not been reported. In this study, we show that miRNA$363-3 \mathrm{p}$ could regulate NOB1 directly and play a critical role in NOB1 expression and inhibit tumor progression in PTC.

\section{MATERIAL AND METHODS}

\section{Prediction of miRNAs targeting NOB1}

TargetScan (http://www.targetscan.org/), miRanda (http:// www.miranda.org/) and PicTar (http://pictar.mdc-berlin. de/) were used to predict miRNAs targeting NOB1 and binding regions.

\section{Cell culture and reagents}

Human PTC cell lines (TPC-1, BCPAP, K1) were obtained from the Cell Bank of the Chinese Academy of Science (Shanghai, China). All cells were cultured with Dulbecco's Modified Eagle Medium (DMEM) containing 10\% fetal bovine serum (FBS), $100 \mathrm{U} / \mathrm{mL}$ penicillin and $100 \mathrm{mg} / \mathrm{mL}$ streptomycin at $37^{\circ} \mathrm{C}$ in humidified air with $5 \% \mathrm{CO}_{2}$.

\section{Cell transfection}

The miR-363-3 p mimic and corresponding negative control (miR-NC) were purchased from GenePharma (Shanghai, China). The full-length NOB1 cDNA was purchased from Integrated Biotech Solutions (Shanghai, China). The NOB1 CDNA was reverse transcribed by PCR and ligated into the pcDNA3.1 mammalian expression vector (Invitrogen). Mimic or plasmids were transfected into cells using Lipofectamine 2000 (Invitrogen) following the manufacturer's instruction and the cells were cultured in $2 \mathrm{~mL}$ of complete DMEM medium for 24 hours.

\section{Reverse transcription real-time PCR}

Total RNA was extracted using Trizol reagent from Life Technologies. RNA $(1 \mu \mathrm{g})$ was used for reverse transcription (RT) reaction to generate cDNA. Real-time PCR was performed using Bio-Rad Ssofast Evagreen supermix reagent with synthesized cDNA as template. PCR primers were designed for detecting human NOB1 gene.

\section{Cell proliferation assay}

Cells were seeded at 5000 cells per well in 96-well plates (5000 cells per well) at 24 hours after transfection. Cell proliferation was measured using the cell counting kit-8 (CCK-8, Dojindo, Kumamoto, Japan) at 24 hours, 48 hours and 72 hours after the cells were seeded. Absorbance was determined at $450 \mathrm{~nm}$ using a microplate spectrophotometer (Thermo Labsystems, Vantaa, Finland). Bromodeoxyuridine (BrdU) assay was also used for cell proliferation measurement. After transfection, thyroid papillary carcinoma cell-1 (TPC-1) cells were given a 2-hour pulse of BrdU (Sigma) at $4 \mathrm{mg} / \mathrm{mL}$. Visualisation of new DNA synthesis was done by indirect immunohistochemistry on adherent cultures using primary anti-BrdU antibody followed by a secondary antibody conjugated with horseradish peroxidase. TMB peroxidase substrate kit (Vector, Burlingame, California, USA) was used for detection. The absorbance rate at $450 \mathrm{~nm}$ was recorded using a 96-well plate reader. The numbers of cells was counted using the Countess II FL automated cell counter (Invitrogen).

\section{Cell migration and invasion assay}

Cell migration was evaluated using a wound healing assay. TPC- 1 cell monolayers were scratched using a sterile $10 \mu \mathrm{l}$ pipette tip and then cells were cultured under standard conditions for 24 hours. Following several washes, recovery of the wound was observed and photographed using an X71 inverted microscope (Olympus, Tokyo, Japan). Quantitative analysis of the scratch area of closure was performed using Image J software. The percentage of wound closure was calculated as follows: ((premigration area - migration area)/premigration area) $\times 100$. Results are representative of three independent experiments performed in triplicate.

The transwell invasion assay was performed to determine cell invasion. The $1 \times 10^{5}$ transfected cells were seeded into the upper chamber of Matrigel-coated inserts with freeserum medium. Medium with $10 \%$ FBS was added to the lowed chamber as chemoattractant. The cells were allowed to invade for 48 hours at $37^{\circ} \mathrm{C}$ with $5 \% \mathrm{CO}_{2}$. Then cells invaded to the lower surface of filter were fixed in $70 \%$ ethanol for $30 \mathrm{~min}$ and stained with $0.1 \%$ crystal violet for $10 \mathrm{~min}$. The number of cells that migrated to the lower side was counted in five randomly selected fields under an X71 inverted microscope (Olympus, Tokyo, Japan).

\section{Cell cycle analysis}

The cell cycle distribution was determined using a fluorescence-activated cell sorting (FACS) flow cytometer (FACSCalibur, Becton Dickinson). Briefly, cells were digested and subsequently fixed in $70 \%$ ethanol at $4^{\circ} \mathrm{C}$ 
Position 217-223 of NOB1 3'UTR 5' ...CUGCCUGCCAAGGAAGUGCAAUU...

hsa-miR-363-3p 3' AUguCUACCUAUgGCACGUUAA

Mutation of miRNA sequence paired with target WT position 217-223 of NOB1 3'UTR $\quad$ 5' ...CUGCCUGCCAAGGAAGUGCAAU... Mut position 217-223 of NOB1 3'UTR $\quad 5$ ' ...CUGCCUGCCAAGGAAGAGACCG

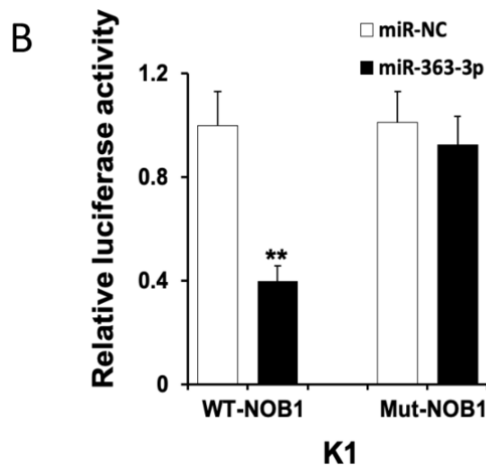

$\mathrm{E}$

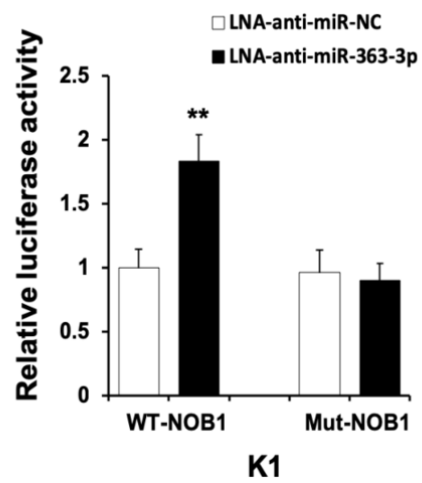

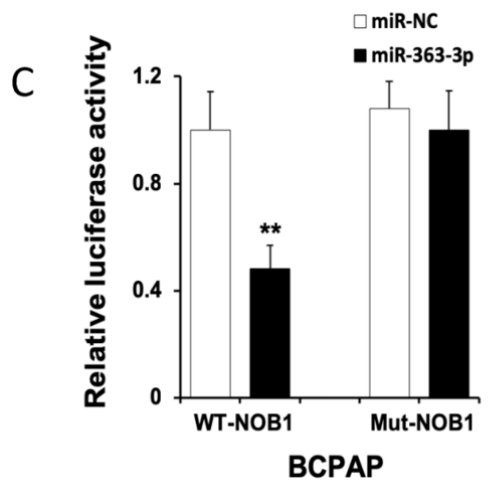

$\mathrm{F}$

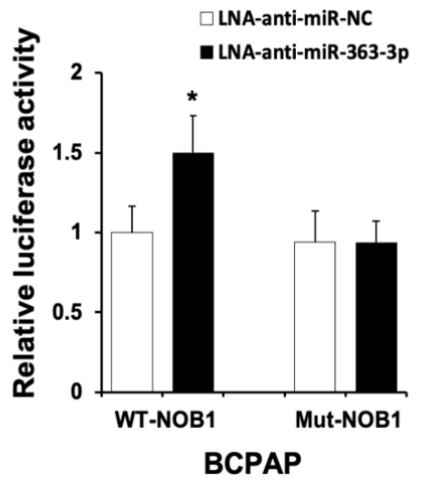

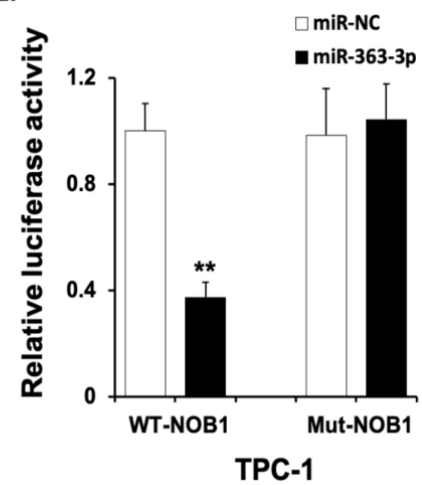

G

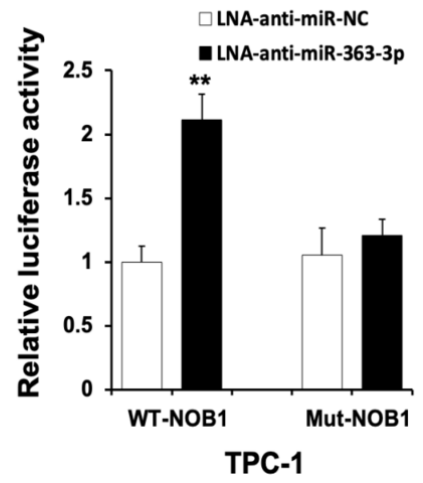

Figure 1 NOB1 targets miR-363-3p. (A) Conservation of the miR-363-3-targeting sites in the NOB1-3'UTR and its mutant sequence that abrogates miR-363-3p binding to target mRNA. The sequence of wild-type (WT) and mutant (mut) miR-363-3p target sites in the NOB1 3'-UTR shown in frame. (B-D). The luciferase reporter assay was used to confirm miR-363-3p targets NOB1. Human papillary thyroid cancer cells (K1, BCPAP, TPC-1) were co-transfected with the miR-363-3 p mimic or miR-NC and NOB1-Wt-3'-UTR or NOB1-Mut-3'UTR reporter plasmid. (E-G) Human papillary thyroid cancer cells (K1, BCPAP, TPC-1) were co-transfected with the LNA- anti-miR-NC or LNAanti-miR-363-3 $p$ and NOB1-Wt-3'-UTR or NOB1-Mut-3'UTR reporter plasmid. All results were derived from independent experiments performed in triplicate $\left({ }^{*} p<0.05,{ }^{* *} p<0.01\right)$.

overnight. Then fixed cells were stained with $50 \mu \mathrm{g} / \mathrm{mL}$ propidium iodide (BD Biosciences) at room temperature for $30 \mathrm{~min}$ in the dark. The cell cycle distribution was analyzed with an FACSCalibur system (BD Biosciences) and ModFit V.3.0 software (BD Biosciences).

\section{Western blot}

Cells were lysed in radioimmunoprecipation assay lysis buffer (Beyotime, Shanghai, China) for $30 \mathrm{~min}$ on ice. After centrifugation at $12,000 \mathrm{~g}, 4^{\circ} \mathrm{C}$ for $10 \mathrm{~min}$, the supernatants were harvested and the protein concentration was quantified using a bicinchoninic acid protein assay kit (Thermo Scientific, Rockford, Illinois, USA) for immunoblotting. A volume of the extract equivalent to $100 \mathrm{mg}$ of total protein was separated on a $10 \%$ sodium dodecyl sulfate-polyacrylamide gel electrophoresis, following by transfer to a polyvinylidene fluoride membrane (Millipore, Billerica, Massachusetts, USA) for immunoblotting. The membranes were blocked in 5\% non-fat milk diluted in TBST (tris-buffered saline, 0.1\% Tween 20) and incubated with the indicated primary antibody: anti-NOB1 (Abcam, Cambridge, UK) and anti- $\beta$-actin antibody (Sigma Aldrich, St. Louis, Missouri, USA). All materials in highest grades uses in the experiments are commercially available.

\section{Tumor xenograft in nude mice}

Twenty female nude mice (BALB/c-null, 6-week-old, $18-20 \mathrm{~g}$ ) were purchased from the Laboratory Animal Center of Jilin University (Changchun, China), and bred in special pathogen-free conditions. All animal procedures were performed in accordance with Institutional Animal Care and Use Committee guidelines of Jilin University (Changchun, China). In $100 \mu \mathrm{l}$ of serum-free DMEM medium, $2 \times 10^{6}$ TPC- 1 cells transfected with NC, miR-NC, miR-363-3 p mimic or Ad/sh-NOB1 (adenovirus expression vector carrying NOB1 siRNA) were harvested, washed, and 
A
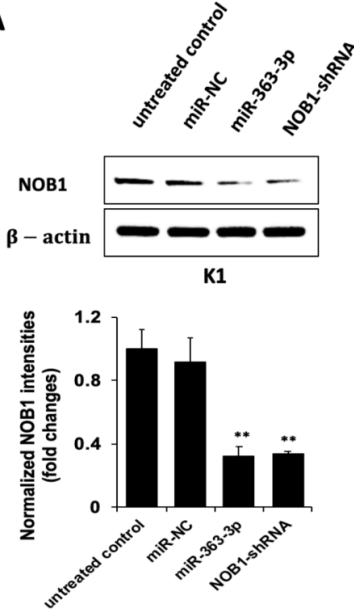

D

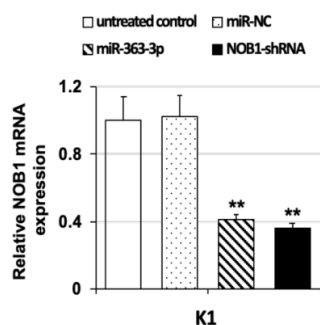

B
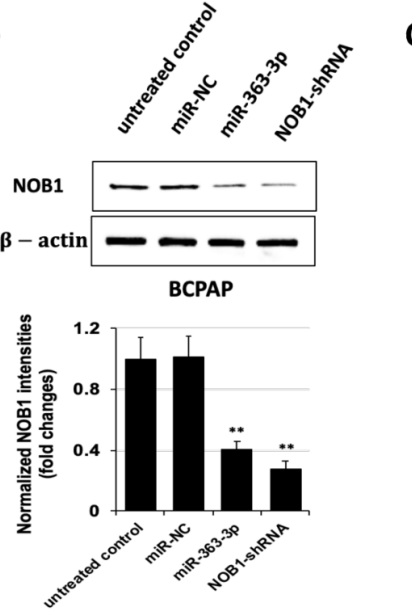

E

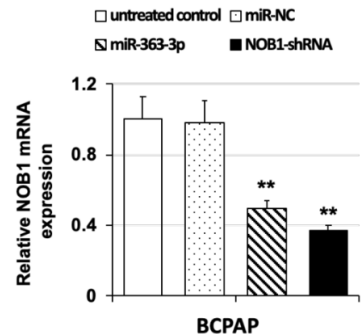

C
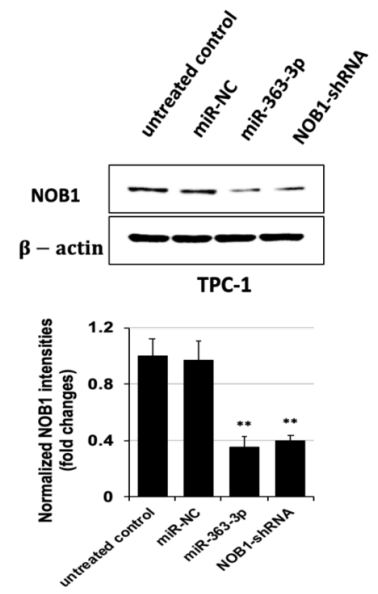

$\mathbf{F}$

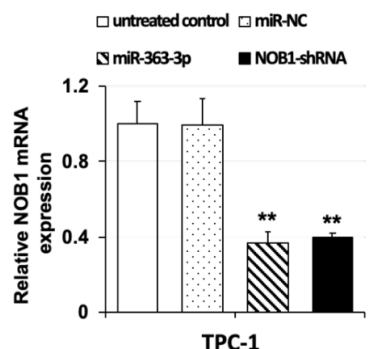

Figure 2 miR-363-3p overexpression downregulates NOB1 protein and mRNA expression. (A-C) Human papillary thyroid cancer cells (K1, BCPAP, TPC-1) were transfected with miR-NC, miR-363-3p or NOB1- shRNA for 48 hours, and then cells lysates were analyzed by immunoblotting with NOB1 and $\beta$-actin antibodies. NOB1 levels were quantified by image J software. $n=3 .{ }^{*} p<0.05,{ }^{* *} p<0.01$, compared with untreated control. Shown are representative blots from three independent experiments. (D-F) qRT-PCR was used to measure NOB1 mRNA expression in human papillary thyroid cancer cells (K1, BCPAP, TPC-1) transfected with miR-NC, miR-363-3 p or NOB1- shRNA for 48 hours. $n=3 .{ }^{* *} p<0.01$, compared with untreated controls.

re-suspended. The cells were then injected subcutaneously into each side of the posterior flank of each nude mouse. Tumor sizes were measured every 5 days from the seventh day of injection. Tumor volume was monitored and calculated according to the formula: $\mathrm{V}=0.5 \times \mathrm{L}$ (length) $\times \mathrm{W}$ (width) by measuring tumor length and width using vernier calipers. Mice were sacrificed 35 days after injection, and tumor tissues were dissected and weighed. MiR-363-3 p, NOB1 and its downstream protein expression were determined in tumor tissues by quantitative real-time (qRT)-PCR and western blot, respectively.

\section{Statistical analysis}

All results were subjected to statistical analysis using oneway or two-way analysis of variance. The data are presented as mean $\pm \mathrm{SD}$. Data were collected from at least three independent experiments, and $\mathrm{p}<0.05$ was considered statistically significant $(* \mathrm{p}<0.05 ; *$ p $<0.01)$.

\section{RESULTS}

\section{NOB1 targets miR-363-3p}

Three computational algorithms including TargetScan, miRanda and PicTar were used in combination to search for miRNAs targeting NOB1 and binding regions. The predicted interaction between miR-363-3 $\mathrm{p}$ and the target site in the NOB1 3'-UTR is illustrated in figure 1A. To explore whether miR-363-3 p targets NOB1 by binding to the 3'-UTR regions, the dual-luciferase reporter vector was constructed containing the 3'-UTR of wild-type NOB1, or its mutant. Non-functional control miR-NC RNAs or hsa-miR-363-3 p RNAs or locked nucleic acid (LNA)-antimiR-NC or LNA-anti-miR-363-3 $p$ were co-transfected with the reporter vectors into the human papillary thyroid cancer cell lines. The miR-363-3p overexpression was shown to significantly decrease the luciferase activity of Wt-NOB1 (wild-type NOB1) while it failed to repress the Mut-NOB1-3'UTR in K1 cells (figure 1B and E), BCPAP cells (figure 1C and F) and TPC-1 cells (figure 1D and G). The dual-luciferase reporter data showed that NOB1 is a target of miR-363-3 p.

\section{MiR-363-3p overexpression downregulates NOB1 protein and mRNA expression}

To investigate if miR-363-3 $\mathrm{p}$ regulates NOB1 protein stability, we transfected human papillary thyroid cancer cell lines (K1, BCPAP and TPC-1 cells) with untreated control, miR-NC, miR-363-3p or NOB1-shRNA. As shown in figure 2A, miR-363-3 p diminished NOB1 levels (up to $-67 \%)$ in $\mathrm{K} 1$ cells compared with negative controls and there were no statistically significant differences between miR-363-3 p overexpression cells and NOB1-shRNA infection cells. As shown in figure $2 \mathrm{~B}, \operatorname{miR}-363-3 \mathrm{p}$ 
A

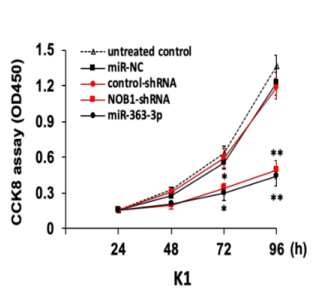

B

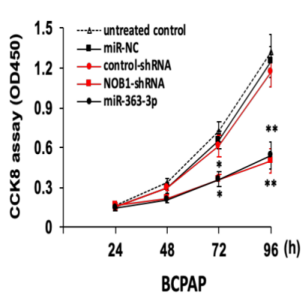

C

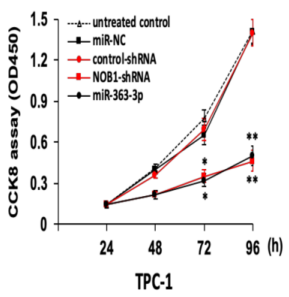

D

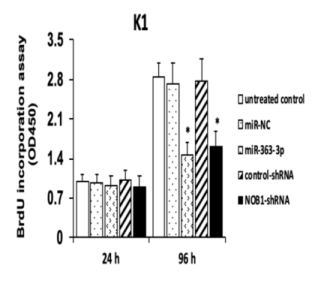

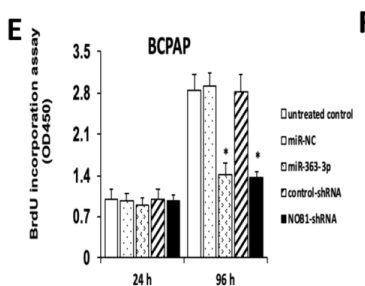
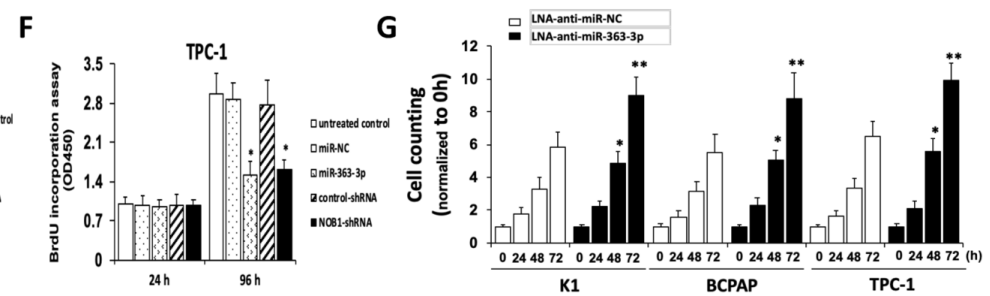

Figure 3 miR-363-3p suppresses the proliferation of papillary thyroid carcinoma (PTC) cells. (A-C) Human papillary thyroid cancer cells (K1, BCPAP, TPC-1) were transfected with miR-NC, control-shRNA, NOB1- shRNA for 24 hours, 48 hours, 72 hours and 96 hours, and the CCK8 assay was performed to measure the cell proliferation. $n=3$. ${ }^{*} p<0.05,{ }^{*} p<0.01$, compared with untreated control. (D-F) Human papillary thyroid cancer cells (K1, BCPAP, TPC-1) were transfected with miR-NC, control-shRNA, NOB1- shRNA for 24 hours and 96 hours, and the BrdU incorporation assay was performed to measure the cells proliferation. $n=3 .{ }^{*} p<0.05,{ }^{* *} p<0.01$, compared with untreated control. (G) Human papillary thyroid cancer cells (K1, BCPAP, TPC-1) were seeded into 6-well plates in triplicates at a density of $5.0 \times 10^{5}$ cells per well, followed by transfection LNA-anti-miR-NC or LNA-anti-miR-363-3p. After 24 hours of culture, the medium was replaced with serum-free medium, and then the cells were cultured for an additional $0-3$ days after transfection. The numbers of cells were counted using the Countess II $\mathrm{fl}$ automated cell counter (Invitrogen). Results are representative of three independent experiments performed in triplicate. ${ }^{*} p<0.05,{ }^{* *} p<0.01$.

diminished NOB1 levels (up to -62\%) in BCPAP cells compared with negative controls. As shown in figure 2C, miR-363-3 p diminished NOB1 levels (up to $-71 \%$ ) in TPC-1 cells compared with negative controls. Further, to examine if the effect of miR-363-3p on the reduction of NOB1 protein mass is due to modulation of NOB1 mRNA expression, we measured NOB1 mRNA levels by RT-realtime PCR in human papillary thyroid cell lines, including $\mathrm{K} 1$ cells (figure 2D), BCPAP cells (figure 2E), TPC-1 cells (figure 2F). MiR-363-3 p overexpression can downregulate NOB1 mRNA expression, suggesting that miR-363-3 p-induced NOB1 reduction is through the regulation of mRNA level.

\section{MiR-363-3p inhibition upregulates NOB1 protein and mRNA expression}

To confirm that miR-363-3p regulates NOB1 protein stability, we transfected human papillary thyroid cancer cell lines (K1, BCPAP and TPC-1 cells) with untreated control, LNA-anti-miR-NC, LNA-anti-miR-363-3 p or pcDNA3.1-NOB1. As shown in online supplemental figure 1, miR-363-3 p significantly repressed NOB1 expression and RNA level in human papillary thyroid cancer cell lines, which was consistent with effects of miR-363-3 p overexpression, suggesting miR-363-3p targets NOB1 to regulate NOB1 stability and RNA interference.

\section{MiR-363-3p suppresses the proliferation of PTC cells}

To determine whether miR-363-3 p regulates PTC proliferation, we ectopically expressed miR-363-3p in the human papillary thyroid cancer cells (K1, BCPAP and TPC-1 cells) by transfecting cells with miR-NC, controlshRNA, NOB1-shRNA or miR-363-3 p on cell proliferation using CCK8 assay. As shown in figure $3 \mathrm{~A}-\mathrm{C}$, ectopic expression of miR-363-3p significantly suppressed the growth of human papillary thyroid cancer cell lines compared with the negative control 72 hours after infection, but there were no statistically significant differences in the growth rate between miR-363-3 p-overexpressed cells and NOB1-shRNA-infected cells. After 96 hours, the growth rate was decreased to over 50\% compared with control cells. There were no significant differences in cell growth between miR-NC cells and the untreated controls. Further, BrdU Incorporation assay was used to confirm the effect of miR-363-3p on the proliferation of human papillary thyroid cancer cells. Similarly, BrdU incorporation was decreased in human papillary thyroid cancer cells after transfection of miR-363-3 p or NOB1-shRNA compared with the controls at 96 hours (figure 3D-F). Further, to examine the effect of miR-363-3 p on regulating cell proliferation, human papillary cancer cells were overexpressed LNA-anti-miR-363-3p or negative control after 24 hours, 48 hours and 72 hours. As shown in figure $3 \mathrm{G}$, suppression of miR-363-3 p significantly increased cell proliferation after 48 hours compared with negative controls through cell counting assay. Taken together, these results suggest that miR-363-3p targets NOB1 as an antagonist to regulate human papillary cancer cells proliferation. 
A
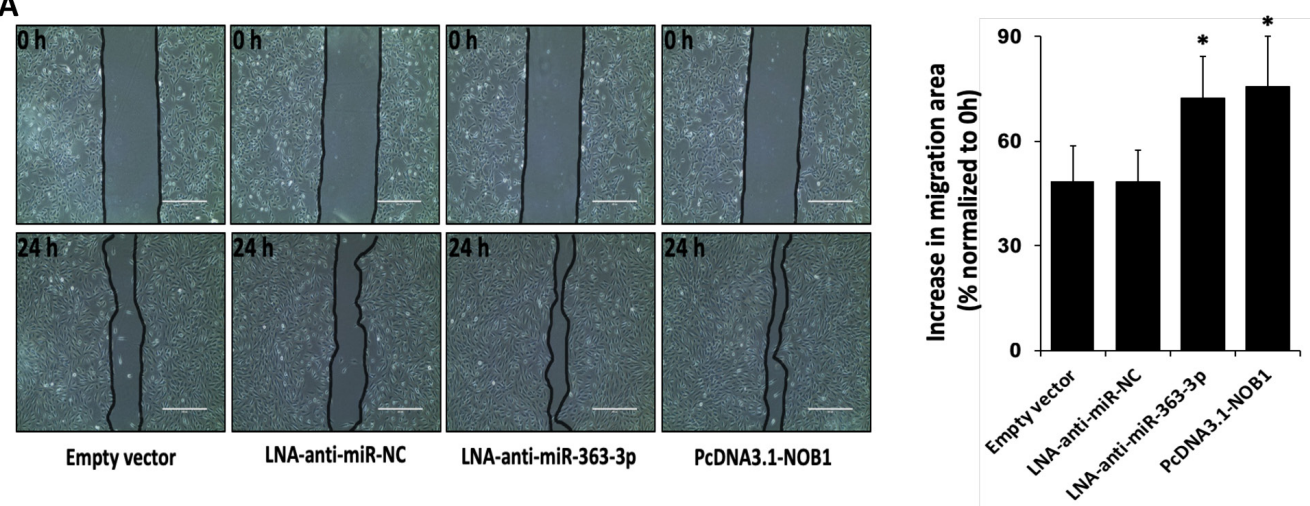

B
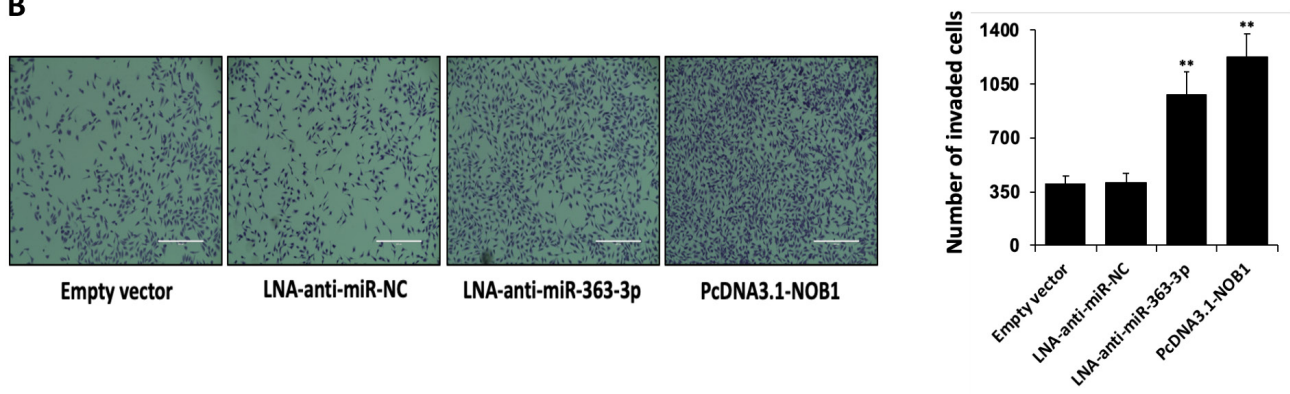

Figure 4 miR-363-3p attenuates the migration and invasion of papillary thyroid carcinoma (PTC) cells. (A) Cell migration was determined by wound healing assay in TPC-1 cells transfected with empty vector, LNA-anti-miR-NC, LNA-anti-miR-363-3p or pcDNA3.1NOB1. (B) Cell invasion was determined by Transwell invasion assay in TPC-1 cells transfected with empty vector, LNA-anti-miR-NC, LNA-anti-miR-363-3 $p$ or pcDNA3.1-NOB1. Results are representative of three independent experiments performed in triplicate. * $p<0.05$, ${ }^{* *} p<0.01$.

\section{MiR-363-3p attenuates the migration and invasion of PTC cells}

Cancer cells migration and invasion are essential for metastasis and prognosis. To investigate if miR-363-3 p regulates cell migration and invasion in human papillary thyroid cancer cells, we examined the effect of inhibition of the expression of miR-363-3 $p$ and overexpression of NOB1 on cell wound healing. TPC- 1 cells were scratched in cell culture dishes and cell migration was observed by measuring the area of wound closure. As shown in figure 4A, inhibition of the expression of miR-363-3 p or overexpression of NOB1 increased cell migration after 24 hours, while the negative controls had no effect. Further, to examine the effect of miR-363-3 p or NOB1 on regulating cell invasion, TPC-1 cells were overexpressed in LNA-anti-miR-363-3 p or pcDNA3.1-NOB1 followed by transwell chamber invasion assay. As shown in figure 4B, inhibition of the expression of miR-363-3 p or overexpression of NOB1 enhanced cell invasive ability significantly compared with the vector control cells. Taken together, these results suggest that miR-363-3 p targets NOB1 as an antagonist to attenuate PTC cell proliferation and invasion.

\section{Effects of MiR-363-3p and NOB1 expression on the cell cycle and apoptosis in PTC cells}

To further investigate the mechanism through which miR-363-3 p or NOB1 inhibits cell proliferation, we determined the effects of miR-363-3p or NOB1 on cell cycle distribution. As shown in figure 5A-C, K1 cells, BCPAP cells or TPC-1 cells transfected with miR-363-3 p or NOB1shRNA showed a promotion of cells in the G0/G1 stage, whereas the numbers of cells in the $S$ phases decreased with flow cytometry assay, which suggested that miR-363-3 p/ NOB1 inhibits cell proliferation by regulating cell cycles. To evaluate the effect of miR-363-3 p or NOB1 on apoptosis, K1 cells, BCPAP cells or TPC-1 cells transfected with miR-363-3 p or NOB1-shRNA followed by staining with Annexin V-FITC and propidium iodide for 72 hours. As shown in figure 5D-F, the miR-363-3p cells or NOB1 silenced cells induced early apoptosis in PTC cells.

\section{MiR-363-3p suppressed tumor growth in nude mice by inhibiting NOB1}

We next evaluated the biological effects of miR-363-3 p on thyroid tumorigenesis in a PTC xenograft mouse model. TPC-1 cells were transfected with miR-NC, miR-363-3 p or NOB1-shRNA and then implanted subcutaneously into nude mice. Tumor growth was evaluated at 7 days for 35 days. Tumor growth curves showed progressive expansion of TPC-1 cells untreated control or transfected with miR-NC, while those transfected with the miR-363-3 p or NOB1-shRNA exhibited slower growth (figure 6A). A significant decrease in the size and weight of the tumors was observed in the miR-363-3 p-overexpression group or NOB1-silence group at 35 days after injection (figure 6B,C). Subsequently, the expression levels of miR-363-3 $\mathrm{p}$ and NOB1 were determined in tumor tissues by RT-PCR and western blot, respectively. We found that tumors from 
A

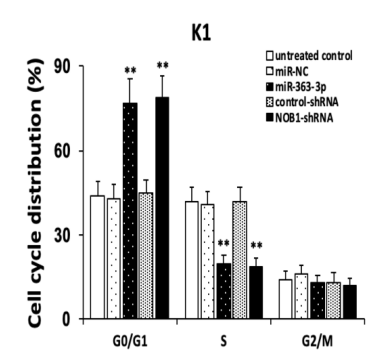

D

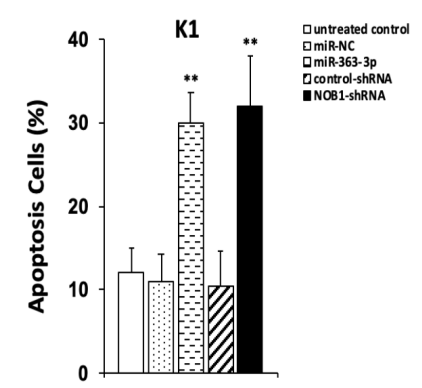

B

C
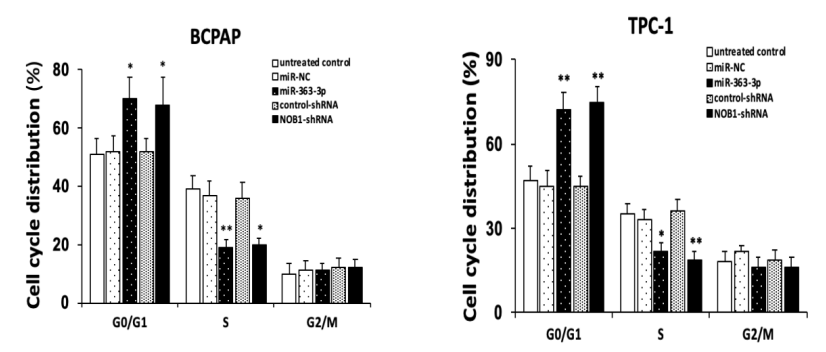

E

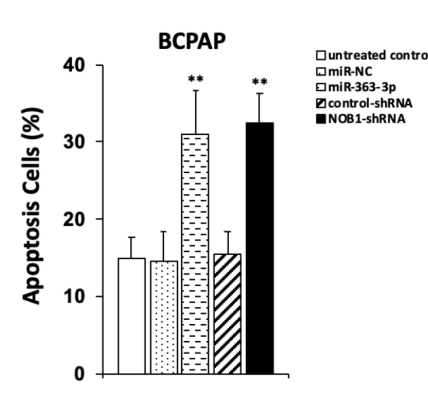

F

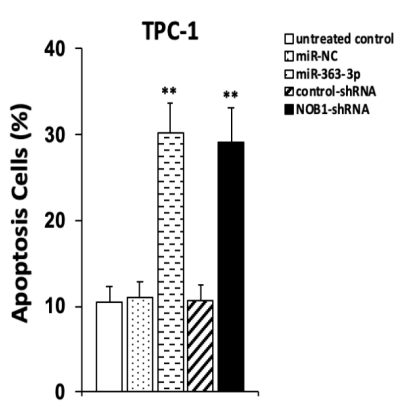

Figure 5 Effects of miR-363-3p and NOB1 expression on the cell cycle and apoptosis in papillary thyroid carcinoma (PTC) cells. (A-C) Human papillary thyroid cancer cells (K1, BCPAP, TPC-1) were transfected with miR-NC, miR-363-3p, control-shRNA or NOB1- shRNA, respectively. After being cultured for 48 hours, cells were fixed and stained with propidium iodide and cell cycle was analyzed by flow cytometry. Results are representative of three independent experiments performed in triplicate. ${ }^{*} p<0.05,{ }^{* *} p<0.01$. (D-F) Human papillary thyroid cancer cells (K1, BCPAP, TPC-1) were transfected with miR-NC, miR-363-3p, control-shRNA or NOB1-shRNA, respectively, followed by staining with annexin V-FITC and propidium iodide for 72 hours. Results are representative of three independent experiments performed in triplicate. ${ }^{*} p<0.05,{ }^{* *} p<0.01$.

A

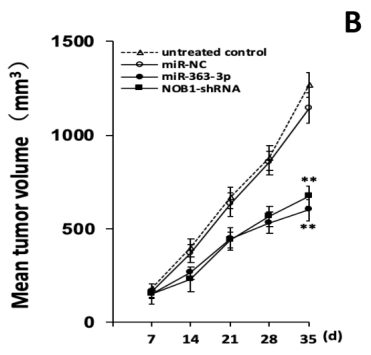

B

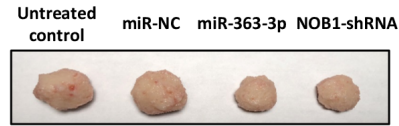

C

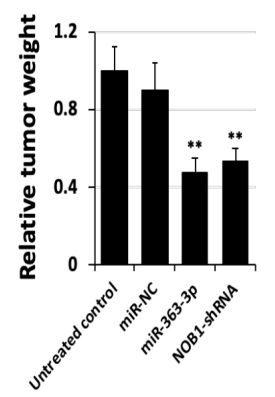

D

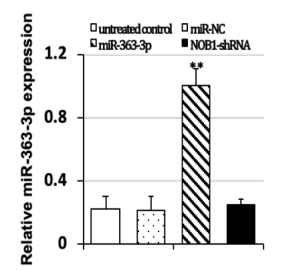

E

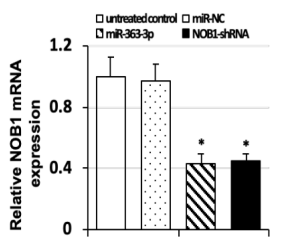

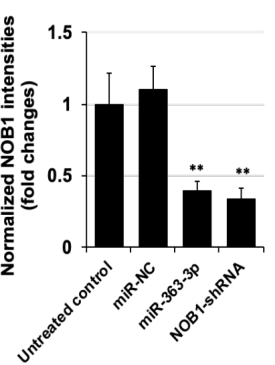

Figure 6 miR-363-3p suppressed tumor growth in nude mice by inhibiting NOB1. (A) Tumor growth curves were established by measuring tumor volume every 7 days for 35 days after injection. (B) Photographs of xenograft tumors from nude mice in each treatment group at 35 days after injection. (C) Tumor weight isolated from nude mice in each treatment group at 35 days after injection. (D) Relative miR-363-3 p expression in xenograft tumors was determined by qRT-PCR. (E) NOB1 protein expression in xenograft tumors was determined by Western blot. NOB1 levels were quantified by image J software. $n=3 .{ }^{*} p<0.05,{ }^{*} p<0.01$, compared with untreated control. Shown are representative blots from three independent experiments. 
the miR-363-3 p-overexpression group showed a significant increase in miR-363-3 p (figure 6D), and a decrease in NOB1 expression (figure 6D,E) compared with the untreated control or miR-NC group.

\section{DISCUSSION}

MicroRNAs (miRNA, miR) are a class of small endogenous non-coding RNA molecules. ${ }^{4}$ These molecules play a crucial role in the regulation of gene expression, through induction of regulation of gene expression or silencing effects by complementary binding to target mRNAs. They are involved in regulating almost all biological process, including cell proliferation, differentiation, apoptosis, hematopoiesis, stress responses and metabolism. MiRNA is also a promising biomarker in many diseases, which has been linked to evaluate cancer progression. ${ }^{30}$ The first published information of the role of miRNA in thyroid tumorigenesis appeared in 2005. ${ }^{31}$ The miRNA involved in PTC initiation, progression and aggressiveness have been studied in serum or tissue. ${ }^{32}$ A large number of studies have demonstrated that different levels of circulating miRNAs are associated with thyroid dysfunction. ${ }^{33}$

Here, we focused on miR-363-3 p, which has been shown to negatively regulate clinical stage and lymph node metastasis and significantly inhibited proliferation, migration and invasion, and tumor growth in PTC cells. Our previous study showed that lower expression of miR-363-3 $p$ was negatively associated with clinical stage and lymph node metastasis and overexpression of miR-363-3 p significantly suppressed PTC growth in vitro and in vivo. Our results suggested that miR-363-3 p functioned as a tumor suppressor in PTC. To date, several targets of miR-363-3 p have been identified, including SOX4, FBW7, myosin 1B, Mcl-1 and S1PR1. On the basis of bioinformatics analysis, we predicted PIK3CA was another miR-363-3 p target to promote tumorigenesis and cancer progression in our previous study. Further, NOB1 has been identified as another target of miR-363-3 p and miR-363-3 p downregulation in papillary thyroid cancer inhibits tumor progression by targeting NOB1.

NOB1 was found to be associated with the $26 \mathrm{~S}$ proteasome and hence plays an essential role in comprehensive biological processes. ${ }^{34}$ Our previous studies showed that NOB1 protein and mRNA expression were significantly upregulated in thyroid carcinoma tissue and downregulation of NOB1 expression significantly inhibited cell proliferation, migration and invasion in PTC cells. Moreover, our previous results also showed that downregulation of NOB1 was able to significantly activate constitutive phosphorylation of p38 MAPK, which might contribute to the inhibition of PTC cell growth. Our findings suggest that NOB1 may be a potential therapeutic target for the treatment of PTC. In this study, we confirmed the results from the bioinformatic tool that miR-363-3 p regulated NOB1 expression at the translational and transcriptional levels by targeting its 3'-UTR. Further, we showed that miR-363-3 p inhibited tumor progression by targeting NOB1 in vitro and in vivo. We found that overexpression of miR-363-3 $p$ or silencing of NOB1 significantly increased G0/G1-phase and decreased $\mathrm{S}$-phase in the human papillary thyroid cells, which led to a significant delay in cell proliferation, indicating miR-363-3 $\mathrm{p}$ and NOB1 are crucial for human papillary thyroid cancer tumorigenesis. Our data unveil that miR-363-3 p negatively regulates NOB1 activity by reducing its stability. This study provides a new therapeutic target for regulation of NOB1 stability to modulate human papillary thyroid cancer progression.

In summary, our current study unveils that miR-363-3 p targets NOB1 and negatively regulates NOB1 in PTC cell lines. Moreover, miR-363-3p downregulation inhibits papillary thyroid cancer cell proliferation, migration and invasion by directly targeting NOB1. Our findings suggest that exogenous overexpression of miR-363-3 p may be a potential drug candidate for the treatment of PTC.

Contributors SD and JL drafted the work and revised it critically for important intellectual content, JL designed the work, YS and ZH did most of the acquisition and analysis for the work, LS and JX did substantial contributions to the interpretation of data for the work.

Funding This study was supported by the National Natural Science Foundation of JILIN Province (202002011449JC), Thyroid Youth Foundation of China, Wu Jieping Foundation of China.

Competing interests None declared.

Patient consent for publication Not required.

Ethics approval The study was approved by the animal experimental ethical committee of the first hospital of Jilin University (20190136).

Provenance and peer review Not commissioned; externally peer reviewed. Data availability statement No data are available.

Supplemental material This content has been supplied by the author(s). It has not been vetted by BMJ Publishing Group Limited (BMJ) and may not have been peer-reviewed. Any opinions or recommendations discussed are solely those of the author(s) and are not endorsed by BMJ. BMJ disclaims all liability and responsibility arising from any reliance placed on the content. Where the content includes any translated material, BMJ does not warrant the accuracy and reliability of the translations (including but not limited to local regulations, clinical guidelines, terminology, drug names and drug dosages), and is not responsible for any error and/or omissions arising from translation and adaptation or otherwise.

Open access This is an open access article distributed in accordance with the Creative Commons Attribution Non Commercial (CC BY-NC 4.0) license, which permits others to distribute, remix, adapt, build upon this work noncommercially, and license their derivative works on different terms, provided the original work is properly cited, an indication of whether changes were made, and the use is non-commercial. See: http://creativecommons.org/ licenses/by-nc/4.0/.

\section{ORCID iD}

Jia Liu http://orcid.org/0000-0002-4528-5199

\section{REFERENCES}

1 Xia Q, Dong S, Bian P-D, et al. Effects of endocrine therapy on the prognosis of elderly patients after surgery for papillary thyroid carcinoma. Eur Arch Otorhinolaryngol 2016;273:1037-43.

2 Liebner DA, Shah MH. Thyroid cancer: pathogenesis and targeted therapy. Ther Adv Endocrinol Metab 2011;2:173-95.

3 Thomas CM, Asa SL, Ezzat S, et al. Diagnosis and pathologic characteristics of medullary thyroid carcinoma-review of current guidelines. Curr Oncol 2019;26:338-44.

4 Bartel DP. Micrornas: genomics, biogenesis, mechanism, and function. Cell 2004;116:281-97.

5 Huntzinger $E$, Izaurralde $E$. Gene silencing by microRNAs: contributions of translational repression and mRNA decay. Nat Rev Genet 2011;12:99-110.

6 Ipsaro JJ, Joshua-Tor L. From guide to target: molecular insights into eukaryotic RNA-interference machinery. Nat Struct Mol Biol 2015;22:20-8.

7 Ding L, Gu H, Xiong X, et al. Micrornas involved in carcinogenesis, prognosis, therapeutic resistance and applications in human triple-negative breast cancer. Cells 2019:8:1492.

8 Lu J, Getz G, Miska EA, et al. Microrna expression profiles classify human cancers. Nature 2005;435:834-8. 
9 Pishkari S, Paryan M, Hashemi M, et al. The role of microRNAs in different types of thyroid carcinoma: a comprehensive analysis to find new miRNA supplementary therapies. J Endocrinol Invest 2018;41:269-83.

10 Chruścik A, Lam AK-yin, Lam AK. Clinical pathological impacts of microRNAs in papillary thyroid carcinoma: a crucial review. Exp Mol Pathol 2015;99:393-8.

11 Sand M, Gambichler T, Sand D, et al. Immunohistochemical expression patterns of the microRNA-processing enzyme Dicer in cutaneous malignant melanomas, benign melanocytic nevi and dysplastic melanocytic nevi. Eur J Dermatol 2011:21:18-21.

12 Hu F, Min J, Cao X, et al. MiR-363-3p inhibits the epithelial-to-mesenchymal transition and suppresses metastasis in colorectal cancer by targeting SOX4. Biochem Biophys Res Commun 2016;474:35-42.

13 Chang J, Gao F, Chu H, et al. miR-363-3p inhibits migration, invasion, and epithelial-mesenchymal transition by targeting NEDD9 and SOX4 in non-smallcell lung cancer. J Cell Physiol 2020;235:1808-20.

14 Zhang R, Li Y, Dong X, et al. Mir-363 sensitizes cisplatin-induced apoptosis targeting in Mcl-1 in breast cancer. Med Oncol 2014;31:347.

15 Chapman BV, Wald Al, Akhtar P, et al. MicroRNA-363 targets myosin 1B to reduce cellular migration in head and neck cancer. BMC Cancer 2015;15:861.

16 Li Y, Chen D, Li Y, et al. Oncogenic cAMP responsive element binding protein 1 is overexpressed upon loss of tumor suppressive miR-10b-5p and miR-363-3p in renal cancer. Oncol Rep 2016;35:1967-78.

17 Zhou P, Huang G, Zhao Y, et al. MicroRNA-363-mediated downregulation of S1PR1 suppresses the proliferation of hepatocellular carcinoma cells. Cell Signal 2014;26:1347-54.

18 Qiao J, Lee S, Paul P, et al. Mir-335 and miR-363 regulation of neuroblastoma tumorigenesis and metastasis. Surgery 2013;154:226-33.

19 Chen Y, Lu X, Wu B, et al. Microrna 363 mediated positive regulation of c-myc translation affect prostate cancer development and progress. Neoplasma 2015;62:191-8.

20 Zhang P-F, Sheng L-L, Wang G, et al. miR-363 promotes proliferation and chemo-resistance of human gastric cancer via targeting of Fbw7 ubiquitin ligase expression. Oncotarget 2016;7:35284-92.

21 Liu J, Li Q, Li R, et al. MicroRNA-363-3p inhibits papillary thyroid carcinoma progression by targeting PIK3CA. Am J Cancer Res 2017;7:148-58.
22 Zhang Y, Ni J, Zhou G, et al. Cloning, expression and characterization of the human Nob1 gene. Mol Biol Rep 2005;32:185-9.

23 Fatica A, Oeffinger M, Dlakić M, et al. Nob1p is required for cleavage of the $3^{\prime}$ end of 18 s rRNA. Mol Cell Biol 2003;23:1798-807.

24 Arcus VL, Bäckbro K, Roos A, et al. Distant structural homology leads to the functional characterization of an archaeal PIN domain as an exonuclease. J Biol Chem 2004;279:16471-8.

25 Lin Y, Peng S, Yu H, et al. Rnai-Mediated downregulation of Nob1 suppresses the growth and colony-formation ability of human ovarian cancer cells. Med Oncol 2012;29:311-7.

26 Che J-P, Li W, Yan Y, et al. Expression and clinical significance of the nin one binding protein and p38 MAPK in prostate carcinoma. Int I Clin Exp Pathol 2013;6:2300-11.

27 Li X-Y, Luo Q-F, Li J, et al. Clinical significance of Nob1 expression in breast infiltrating ductal carcinoma. Int J Clin Exp Pathol 2013;6:2137-44.

28 Li Y, Ma C, Qian M, et al. Downregulation of Nob1 suppresses the proliferation and tumor growth of non-small cell lung cancer in vitro and in vivo. Oncol Rep 2014;31:1271-6.

29 Meng W, Wang P-S, Liu J, et al. Adenovirus-Mediated siRNA targeting Nob1 inhibits tumor growth and enhances radiosensitivity of human papillary thyroid carcinoma in vitro and in vivo. Oncol Rep 2014;32:2411-20.

30 Zhang B, Pan X, Cobb GP, et al. microRNAs as oncogenes and tumor suppressors. Dev Biol 2007;302:1-12.

$31 \mathrm{He} \mathrm{H}$, Jazdzewski K, Li W, et al. The role of microRNA genes in papillary thyroid carcinoma. Proc Natl Acad Sci U S A 2005;102:19075-80.

32 Zembska A, Jawiarczyk-Przybyłowska A, Wojtczak B, et al. Microrna expression in the progression and aggressiveness of papillary thyroid carcinoma. Anticancer Res 2019;39:33-40.

33 Yoruker EE, Terzioglu D, Teksoz S, et al. Microrna expression profiles in papillary thyroid carcinoma, benign thyroid nodules and healthy controls. J Cancer 2016;7:803-9.

34 Veith T, Martin R, Wurm JP, et al. Structural and functional analysis of the archaeal endonuclease Nob1. Nucleic Acids Res 2012;40:3259-74. 